Case Report

\title{
Prolonged Response of a Patient with Relapsed Acute Myeloid Leukemia to a Novel Oral Bromodomain Extraterminal Inhibitor (BETi)
}

\author{
Meilen Chang Muñoz, ${ }^{1}$ Jennifer A. Murphy, ${ }^{2,3}$ Johannes E. Wolff, ${ }^{4,}$ and Brian A. Jonas ${ }^{1,3}$ \\ ${ }^{1}$ Department of Internal Medicine, University of California Davis, School of Medicine, Sacramento, CA, USA \\ ${ }^{2}$ Department of Pharmacy Services, University of California Davis Medical Center, Sacramento, CA, USA \\ ${ }^{3}$ University of California Davis Comprehensive Cancer Center, Sacramento, CA, USA \\ ${ }^{4}$ AbbVie Inc., North Chicago, IL, USA \\ Correspondence should be addressed to Brian A. Jonas; bajonas@ucdavis.edu
}

Received 11 May 2020; Revised 18 November 2020; Accepted 30 November 2020; Published 16 December 2020

Academic Editor: Kostas Konstantopoulos

Copyright ( 92020 Meilen Chang Muñoz et al. This is an open access article distributed under the Creative Commons Attribution License, which permits unrestricted use, distribution, and reproduction in any medium, provided the original work is properly cited.

\begin{abstract}
Acute myeloid leukemia (AML) is an aggressive clonal bone marrow cancer characterized by high rates of relapse and mortality. A middle-aged woman with AML relapsed twice after achieving complete remission with induction therapy and subsequent salvage therapy. She was then enrolled in a clinical trial with the bromodomain extraterminal inhibitor (BETi) mivebresib and achieved complete remission with incomplete count recovery (CRi) with monotherapy. Subsequently, she relapsed and was transitioned to combination therapy with mivebresib plus venetoclax and achieved CR again. The patient required eltrombopag to decrease platelet dependence in both arms of the trial and exhibited less myelosuppression with the combination therapy. The exceptional response to mivebresib demonstrated by this patient underscores the therapeutic potential of mivebresib.
\end{abstract}

\section{Introduction}

Acute myeloid leukemia (AML) is an aggressive and heterogeneous bone marrow cancer driven by genetic and epigenetic alterations. It is the second most common type of adult leukemia making up approximately $30 \%$ of all cases and is the leading cause of leukemia-related deaths $[1,2]$. Risk factors for development of AML include age $>65$, male gender, smoking, prior treatment with chemotherapy or radiation, certain environmental exposures, and history of antecedent myeloid disorder, such as myelodysplastic syndrome $[3,4]$. The 5 -year overall survival rate ranges from $40-50 \%$ in younger patients to $20-30 \%$ in older patients who receive high-intensity chemotherapy [5]. Relapse rates vary from $30-35 \%$ in patients aged $<60$ with favorable risk factors, up to $90 \%$ in patients aged $>60$ with adverse risk factors [6]. If the disease relapses, salvage therapy may be offered to achieve remission again, but survival in the relapsed setting is very poor [7]. Given the poor outcomes seen with AML, especially in the relapsed setting, it is imperative to find new strategies to improve remission and overall survival rates.

Bromodomain (BRD) and extraterminal (BET) proteins are a family of proteins that recognize acetylated lysine residues on histones to promote chromosome remodeling, histone modification or recognition and control transcription machinery to upregulate gene expression driving oncogenesis $[8,9]$. Preclinical studies demonstrate that BET inhibitors (BETis) have antiproliferative activity and trigger a strong apoptotic response in cell lines derived from hematologic malignancies through the downregulation of MYC [10]. Thus, BET proteins have been identified as key targets for leukemia therapy $[11,12]$. Mivebresib is a potent oral BETi that has recently entered phase I clinical trials [13]. It functions in a variety of mechanisms including G1 cell cycle arrest, induction of apoptosis, and potentially targeting 


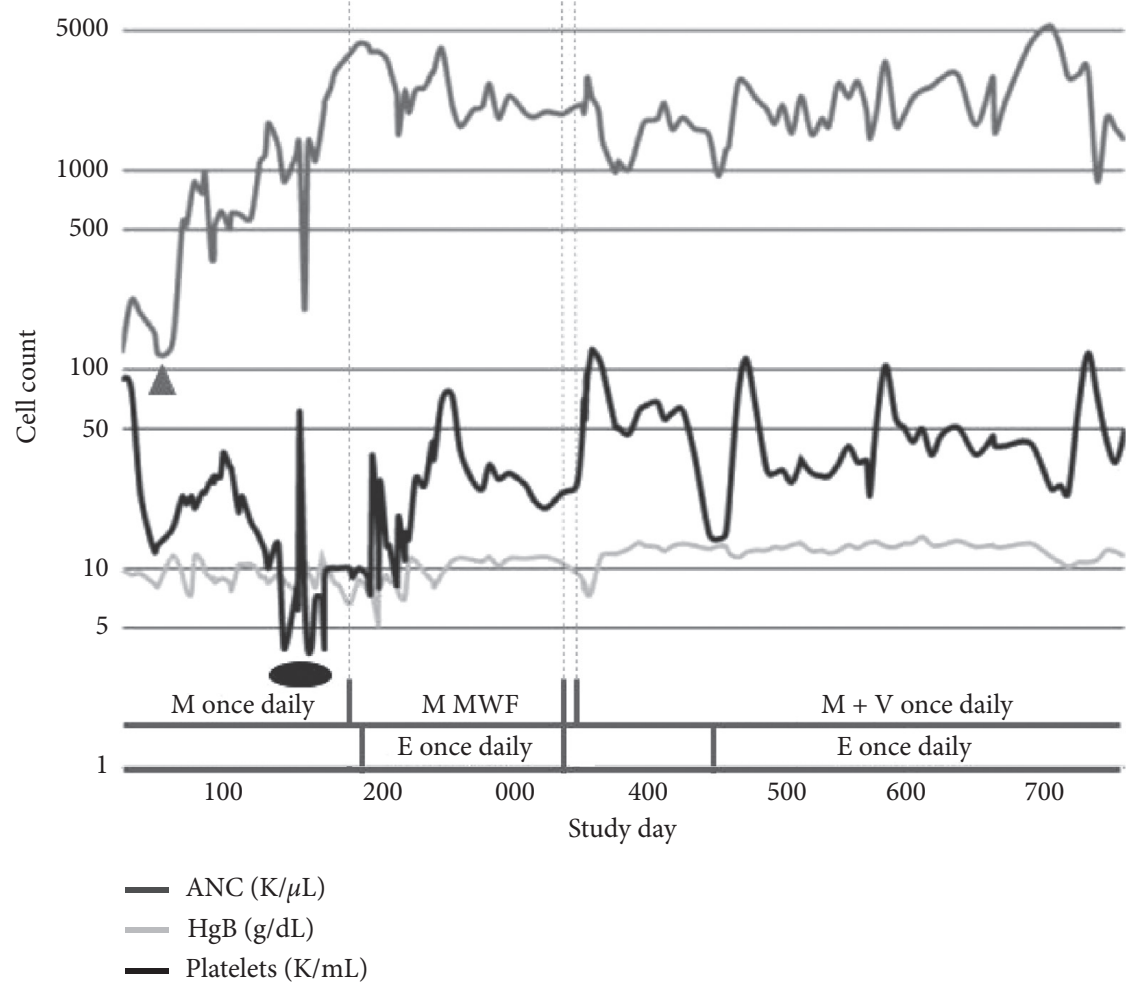

FIGURE 1: Absolute neutrophil count (ANC), hemoglobin ( $\mathrm{Hgb}$ ), and platelet counts throughout mivebresib mono- or combination therapy with venetoclax. ANC was less than $100 / \mu \mathrm{L}$ during days 1 to 84 , corresponding to cycles 1 to 3 of monotherapy (as denoted by grey triangle), and recovered to greater than $1,000 / \mu \mathrm{L}$, thereafter. Hgb generally remained between 7 and $12 \mathrm{~g} / \mathrm{dL}$ throughout all treatment modalities. Platelets remained less than $10,000 / \mu \mathrm{L}$ between days 140 and 190, corresponding to cycles 4 and 6 of monotherapy (as denoted by black oval), and then increased to 15,000 to $120,000 / \mu \mathrm{L}$ for the duration of the trial. $\mathrm{M}=$ mivebresib; $\mathrm{V}=$ venetoclax; $\mathrm{E}=$ eltrombopag.

the tumor microenvironment to provide additional therapeutic benefit. Another agent that has demonstrated high response rates and encouraging remission durations in AML is venetoclax, an oral B-cell lymphoma-2 (BCL-2) inhibitor that can activate apoptosis in malignancies [14]. Venetoclax is approved in combination with a hypomethylating agent (HMA) or low-dose cytarabine (LDAC) backbone in older patients with newly diagnosed AML or those who are not candidates for intensive chemotherapy $[15,16]$.

Like other systemic therapies, mivebresib and venetoclax cause myelosuppression that can lead to transfusion dependence. Allogeneic blood transfusions are associated with transfusion-related organ injury and sepsis, hemolytic transfusion reactions [17], platelet-refractory alloimmunization [18], and increased graft-versus-host disease after allogeneic hematopoietic stem cell transplantation [19] and hinder finding a suitable stem cell donor. Hence, bone marrow growth factors have been used to promote blood count recovery and decrease transfusion dependency. For example, eltrombopag is a potent thrombopoietin receptor agonist that has been studied for use in AML among other diseases characterized by thrombocytopenia [20].

In this study, we report the exceptional clinical response of a patient with multiply relapsed AML who was treated with mivebresib as monotherapy and subsequently after relapse with mivebresib in combination with venetoclax and eltrombopag. Complete remission with incomplete count recovery (CRi) was achieved with monotherapy, and after relapse she achieved a second $\mathrm{CRi}$ with combination therapy.

\section{Case Description}

A 60-year-old woman with no significant past medical history presented with de novo AML discovered on routine CBC. She presented with night sweats, bruising, and pancytopenia. The initial bone marrow biopsy revealed 66\% myeloblasts, $30-40 \%$ cellularity, normal karyotype, and negative molecular studies. She achieved complete remission (CR) with $7+3$ induction therapy and proceeded with 4 cycles of high-dose cytarabine consolidation. She remained in remission for nearly 20 months when a surveillance bone marrow biopsy revealed relapsed disease. She subsequently underwent salvage reinduction and achieved a second CR. The patient declined allogenic hematopoietic stem cell transplant in favor of consolidation therapy with intermediate-dose cytarabine. She remained in CR for approximately 10 months without additional therapy before relapsing for a second time.

The patient subsequently elected to enroll in a phase I clinical trial of mivebresib for relapsed or refractory AML (NCT02391480) and was treated with mivebresib monotherapy. Her disease did not initially respond with an increase in marrow blasts from $21 \%$ to $40 \%$ after the first cycle, 
TABLE 1: Clinical course, including bone marrow, molecular, and peripheral blood responses.

\begin{tabular}{|c|c|c|c|c|c|c|c|c|c|c|}
\hline Day & $\begin{array}{l}\text { Circulating } \\
\text { blasts (\%) }\end{array}$ & $\begin{array}{l}\text { BM blast } \\
\text { morphology } \\
(\%)\end{array}$ & $\begin{array}{c}\text { MLFS } \\
\text { achieved }\end{array}$ & $\begin{array}{c}\text { MRD } \\
\text { flow } \\
\text { blasts } \\
(\%) \\
\end{array}$ & Cytogenetics & Molecular & $\begin{array}{c}\text { ANC } \\
(\mathrm{K} / \mu \mathrm{L})\end{array}$ & $\begin{array}{l}\mathrm{Hgb} \\
(\mathrm{g} / \mathrm{dL})\end{array}$ & $\begin{array}{c}\text { Plt } \\
(\mathrm{K} / \mu \mathrm{L})\end{array}$ & ELN response \\
\hline $\begin{array}{l}\text { Screening } \\
\text { mono (M) }\end{array}$ & 6 & 21 & N/A & $\begin{array}{l}\text { Not } \\
\text { done }\end{array}$ & 46,XX [20] & $\begin{array}{c}\text { STAG2 } \\
\text { R213Efster12 } \\
\text { (VAF 18\%) }\end{array}$ & 0.12 & 10.6 & 95 & N/A \\
\hline $\mathrm{C} 2 \mathrm{D} 1 \mathrm{M}$ & 3 & 40 & No & $\begin{array}{l}\text { Not } \\
\text { done }\end{array}$ & 46,XX [18] & Not done & 0.12 & 8 & 13 & $\begin{array}{l}\text { Resistant } \\
\text { disease }\end{array}$ \\
\hline $\mathrm{C} 3 \mathrm{D} 1 \mathrm{M}$ & 0 & 11 & No & $\begin{array}{l}\text { Not } \\
\text { done }\end{array}$ & Failed & Not done & 0.76 & 7.5 & 23 & $\begin{array}{l}\text { Resistant } \\
\text { disease }\end{array}$ \\
\hline C5D1M & 0 & 8 & No & $\begin{array}{l}\text { Not } \\
\text { done }\end{array}$ & 46,XX [20] & Not done & 1.2 & 9 & 12 & $\begin{array}{l}\text { Resistant } \\
\text { disease }\end{array}$ \\
\hline C6D1M & 0 & 4 & Yes & 20.1 & $46, X X[20]$ & Not done & 1.34 & 8.9 & 4 & CRi \\
\hline C7D1M & 0 & 4 & Yes & 10.8 & $46, X X[20]$ & Not done & 3.3 & 8.2 & 10 & $\mathrm{CRi}$ \\
\hline C9D1M & 0 & 8 & No & 12.1 & 46,XX [20] & Not done & 2.5 & 10.9 & 28 & $\begin{array}{l}\text { Morphologic } \\
\text { relapse }\end{array}$ \\
\hline C10D1M & 0 & 4 & Yes & $\begin{array}{l}\text { Not } \\
\text { done }\end{array}$ & 46,XX [20] & Not done & 3.1 & 8.6 & 43 & CRi \\
\hline C11D1M & 0 & 8 & No & 13.5 & $46, \mathrm{XX}[20]$ & $\begin{array}{c}\text { STAG2 } \\
\text { R213Efster12 } \\
\text { (VAF 6.6\%), } \\
\text { WT1 K467Ter } \\
\text { (VAF 7.4\%) }\end{array}$ & 2.7 & 11.4 & 33 & $\begin{array}{l}\text { Morphologic } \\
\text { relapse }\end{array}$ \\
\hline C13D1M & 0 & 14 & No & 26.3 & 46,XX [20] & Not done & 1.9 & 10.6 & 24 & $\begin{array}{l}\text { Resistant } \\
\text { disease }\end{array}$ \\
\hline $\begin{array}{l}\text { Screening } \\
\text { combo }(\mathrm{C})\end{array}$ & $\begin{array}{c}\text { Not } \\
\text { reported }\end{array}$ & 8 & N/A & $\begin{array}{l}\text { Not } \\
\text { done }\end{array}$ & $46, \mathrm{XX}[20]$ & Not done & $>1.0$ & 9.3 & 29 & $\mathrm{~N} / \mathrm{A}$ \\
\hline $\mathrm{C} 2 \mathrm{D} 1 \mathrm{C}$ & 0 & 2 & Yes & 0.99 & $46, \mathrm{XX}[20]$ & Not done & 1.1 & 11.9 & 51 & CRi \\
\hline C3D1C & 0 & 2 & Yes & 0.64 & $46, \mathrm{XX}[20]$ & Not done & 1.8 & 12.8 & 68 & CRi \\
\hline C5D1C & 0 & 1 & Yes & 0.35 & $46, X X[20]$ & None detected & 1.3 & 12 & 20 & CRi \\
\hline C7D1C & 0 & 2 & Yes & 0.14 & $46, X X[20]$ & Not done & 1.5 & 12.2 & 30 & CRi \\
\hline C9D1C & 0 & 1 & Yes & 0.34 & 46,XX [20] & Not done & 3.5 & 12.9 & 104 & CR \\
\hline C11D1C & 0 & N/A & N/A & 0.9 & $46, X X[20]$ & None detected & 3 & 13.7 & 51 & CRi \\
\hline C13D1C & 0 & 6 & No & 5.3 & $46, X X[20]$ & Not done & 4.4 & 13.3 & 42 & $\begin{array}{l}\text { Morphologic } \\
\text { relapse }\end{array}$ \\
\hline C14D1C & 0 & 4 & Yes & 5.5 & 46,XX [20] & $\begin{array}{c}\text { KMT2A I3423V } \\
\text { (VUS, VAF } \\
50.6 \%)\end{array}$ & 2.9 & 10.2 & 24 & CRi \\
\hline C15D1C & 0 & 10 & No & 10.7 & 46,XX [20] & Not done & 1.4 & 11.5 & 50 & $\begin{array}{l}\text { Morphologic } \\
\text { relapse }\end{array}$ \\
\hline $\begin{array}{l}\text { EOT } \\
\text { marrow } \\
\text { deferred }\end{array}$ & & & & & & & 2.6 & 11.7 & 75 & \\
\hline
\end{tabular}

BM, bone marrow; MLFS, morphologic leukemia-free state; MRD, measurable residual disease; VAF, variant allele frequency; ANC, absolute neutrophil count; Hgb, hemoglobin; Plt, platelet; ELN, European LeukemiaNet; CRi, complete remission with incomplete count recovery; CR, complete remission; N/A, not applicable; EOT, end of treatment; VUS, variant of uncertain significance.

but this was followed by a decrease to $11 \%$ after cycle 2 and eventual CRi, due to ongoing thrombocytopenia, by the end of cycle 5. The patient first developed thrombocytopenia during cycle 2 and became transfusion dependent by cycle 5 (Figure 1 and Table 1). Eltrombopag $50 \mathrm{mg}$ daily was added during cycle 7 , and mivebresib was modified from $1.5 \mathrm{mg}$ daily to $3 \mathrm{mg}$ MWF during cycle 8 resulting in decreased transfusion requirements (Figure 2). Eltrombopag was increased to $100 \mathrm{mg}$ daily, eliminating transfusion dependency, but the patient remained thrombocytopenic. Prior to the start of cycle 13 of mivebresib monotherapy, the patient relapsed with $14 \%$ blasts. She then discontinued mivebresib monotherapy and, after a washout period, was enrolled in a drug combination cohort of the study. The patient initially achieved CRi after cycle 1 of treatment with mivebresib and venetoclax (target dose $800 \mathrm{mg}$ ) before improving to CR after cycle 8 (Table 1). Eltrombopag was held during the early cycles of combination therapy as she maintained a platelet count greater than $50,000 / \mu \mathrm{L}$. However, during cycle 4 of mivebresib and venetoclax, the patient developed leukopenia and thrombocytopenia and eltrombopag was resumed. Platelet counts remained greater than $20,000 / \mu \mathrm{L}$ and eventually improved to greater than $100,000 / \mu \mathrm{L}$ with eltrombopag, mivebresib, and venetoclax. In contrast, 


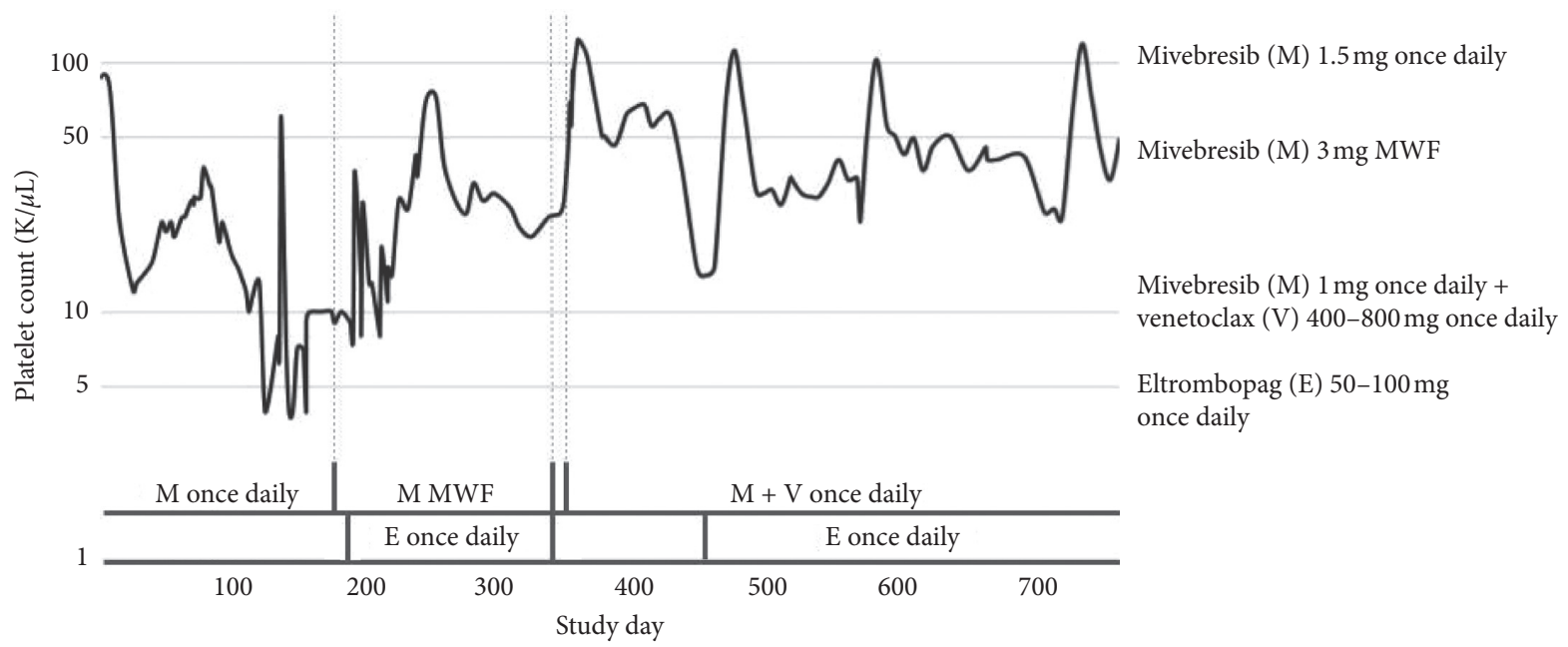

Figure 2: Platelet count response to therapy. Platelets decreased to less than $10,000 / \mu \mathrm{L}$ with mivebresib $1.5 \mathrm{mg}$ once daily monotherapy. Platelets increased to greater than $10,000 / \mu \mathrm{L}$ with a change in mivebresib dosing to $3 \mathrm{mg} \mathrm{MWF}$ and increased further to greater than $50,000 /$ $\mu \mathrm{L}$ with the addition of eltrombopag. Platelet counts remained greater than $15,000 / \mu \mathrm{L}$ and up to $120,000 / \mu \mathrm{L}$ with the combination mivebresib and venetoclax and sustained with eltrombopag.

platelet counts generally had previously remained below $30,000 / \mu \mathrm{L}$ with eltrombopag and mivebresib monotherapy.

Pretrial genomic screening revealed a STAG2 R213Efster12 mutation with $18 \%$ variant allele frequency (VAF) (Table 1). Genomics performed during cycle 11 of monotherapy revealed persistence of STAG2 mutation with a decrease in VAF to $6.6 \%$ and a new mutation WT1 K467Ter (VAF 7.4\%). Genomics performed during cycle 5 of combination therapy did not detect any mutations. However, a KMT2A I3432V (VAF 50.6\%) variant of unknown significance was detected after cycle 13, and the patient relapsed soon thereafter.

\section{Discussion}

AML comprises $\sim 30 \%$ of all adult leukemia cases, and relapse rates can be as high $90 \%$ in elderly patients with adverse risk factors. High relapse rates necessitate novel systemic therapies with longer remission durations. New targets have been identified that can induce apoptosis in malignant cells, including BET and BCL-2 proteins. Mivebresib is a pan-inhibitor of the BET proteins that is currently in phase I clinical trials. In this case, mivebresib was initially used as a monotherapy per protocol with the patient achieving CRi after completion of cycle 5. Given that the patient continued to experience severe thrombocytopenia and transfusion dependence, which prevented the patient from achieving CR, as defined by $<5 \%$ blasts by morphology in a cellular marrow with recovery of both neutrophils and platelets, eltrombopag was added as a supportive measure. This resulted in decreased transfusion dependency, but the thrombocytopenia persisted. At disease relapse, mivebresib monotherapy was discontinued, and after a washout period, combination therapy with venetoclax and mivebresib was started. The patient once again achieved CRi during cycle 2 before improving to a CR after cycle 8 . Response was maintained for approximately 11 months until cycle 13 when there was morphologic relapse.

Interestingly, platelet counts were not as low with combination therapy as with monotherapy, and the patient sustained only mild to moderate thrombocytopenia with the addition of eltrombopag to venetoclax and mivebresib. She did not require any platelet transfusions with this combination regimen. Possible explanations for this observation include a deeper response to the combination therapy resulting in improved count recovery, response related to the venetoclax component alone, or that combination therapy may have resulted in a more effective response to eltrombopag potentially attributable to a synergistic effect between the three drugs.

Studies demonstrate that eltrombopag stimulates thrombopoiesis via the JAK-STAT signaling cascade [21]. This same pathway regulates expression of the apoptosis regulatory genes BCL-2 [22] and MYC [23]. Given that venetoclax is a BCL-2 inhibitor and mivebresib downregulates MYC expression, we can hypothesize that venetoclax and mivebresib downregulate the apoptotic pathway in platelets, allowing the cell proliferation pathway to predominate. Additional studies are required to explore these potential mechanisms of response and synergy.

In addition, we can consider the contribution of STAG2 deficiency on incomplete count recovery. STAG2 is a part of the cohesin multimeric protein complex responsible for cohesion of sister chromatids, transcription regulation, and homologous DNA repair [24]. Preliminary data suggest that loss of STAG2 can lead to dysregulated transcription in AML [25]. Considering that our patient initially expressed a STAG2 mutation, it is conceivable that STAG2 deficiency globally prevented appropriate transcription, inevitably inhibiting effective platelet production. Once this mutation resolved in the setting of combination therapy, we posit that eltrombopag-induced platelet production was more efficient. Given resolution of this mutation with combination 
therapy, we can also consider the potential for STAG2 to be a predictive marker for response to mivebresib-based therapy.

\section{Conclusion}

In this case study of an exceptional responder, mivebresib as monotherapy and in combination with venetoclax conferred an 18-month survival supporting the potential clinical activity of this novel BETi in relapsed or refractory AML. In combination with mivebresib and/or venetoclax, eltrombopag also led to decreased transfusion dependence, which can augment quality of life, decrease healthcare costs, and reduce platelet alloimmunization. Primary data analyses of all patients in the AML cohorts investigated in this trial, including safety and clinical efficacy, will be described elsewhere.

\section{Data Availability}

The data supporting the conclusions for this study are confidential and protected by under HIPAA Privacy Rule compliance measures.

\section{Conflicts of Interest}

Meilen Chang Muñoz has no conflicts of interest to disclose. Jennifer Murphy: owning shares in Gilead; research funding to her institution from Novartis. Johannes Wolff: AbbVie employee and may own stock; travel, accommodations, and expenses from AbbVie. Brian A. Jonas: consulting/advising for AbbVie, Amgen, Celgene, GlycoMimetics, Jazz, Pharmacyclics, Takeda, Tolero, and Treadwell; travel reimbursement from AbbVie, Amgen, and GlycoMimetics; research funding to his institution from AbbVie, Accelerated Medical Diagnostics, AROG, Celgene, Daiichi Sankyo, F. Hoffmann-La Roche, Forma, Genentech/Roche, GlycoMimetics, Hanmi, Incyte, Jazz, LP Therapeutics, Pfizer, Pharmacyclics, and Sigma Tau.

\section{Authors' Contributions}

MCM, JAM, JW, and BAJ were responsible for conception and design; MCM, JAM, and BAJ were involved in analysis and interpretation of data; MCM, JAM, JW, and BAJ were responsible for writing, review, and/or revision of the manuscript.

\section{Acknowledgments}

The authors wish to thank our patient for participating in the AbbVie-sponsored clinical trial (NCT02391480) and Nichole Mahaffey, $\mathrm{PhD}$, for assistance with the data. The trial was approved by the UC Davis Institutional Review Board (\#984817). This case study did not have funding support. The case belongs to a clinical study (NCT02391480), and the case is eligible to be published as an isolated case report. The clinical trial the patient participated in was funded by AbbVie.

\section{References}

[1] A. Redaelli, J. M. Lee, J. M. Stephens, and C. L. Pashos, "Epidemiology and clinical burden of acute myeloid leukemia," Expert Review of Anticancer Therapy, vol. 3, no. 5, pp. 695-710, 2003.

[2] R. L. Siegel, K. D. Miller, and A. Jemal, "Cancer statistics, 2020," CA: A Cancer Journal for Clinicians, vol. 70, no. 1, pp. 7-30, 2020.

[3] S. A. Speer, J. C. Semenza, T. Kurosaki et al., "Risk factors for acute myeloid leukemia and multiple myeloma: a combination of GIC and case-control studies," Journal of Environmental Health, vol. 64, no. 7, pp. 8-15, 2002.

[4] S. S. Strom, R. Oum, K. Y. Elhor Gbito, G. Garcia-Manero, and Y. Yamamura, "De novo acute myeloid leukemia risk factors," Cancer, vol. 118, no. 18, pp. 4589-4596, 2012.

[5] H. Kantarjian, "Acute myeloid leukemia-major progress over four decades and glimpses into the future," American Journal of Hematology, vol. 91, no. 1, pp. 131-145, 2016.

[6] C. Röllig, M. Bornhäuser, C. Thiede et al., "Long-term prognosis of acute myeloid leukemia according to the new genetic risk classification of the European leukemianet recommendations: evaluation of the proposed reporting system," Journal of Clinical Oncology, vol. 29, no. 20, pp. 2758-2765, 2011.

[7] D. A. Breems, W. L. J. Van Putten, P. C. Huijgens et al., "Prognostic index for adult patients with acute myeloid leukemia in first relapse," Journal of Clinical Oncology, vol. 23, no. 9, pp. 1969-1978, 2005.

[8] G. Borthakur, J. E. Wolff, I. Aldoss et al., "First-in-human study of ABBV-075 (mivebresib), a pan-inhibitor of bromodomain and extra terminal (BET) proteins, in patients (pts) with relapsed/refractory (RR) acute myeloid leukemia (AML): preliminary data," Journal of Clinical Oncology, vol. 36, no. 15, p. 7019, 2018.

[9] G. M. Borthakur, O. Odenike, I. Aldoss et al., "Biomarker modulation by mivebresib (ABBV-075) +/- venetoclax in relapsed/refractory acute myeloid leukemia," Blood, vol. 134, no. 1 , p. 539, 2019.

[10] M. H. Bui, X. Lin, D. H. Albert et al., "Preclinical characterization of BET family bromodomain inhibitor ABBV-075 suggests combination therapeutic strategies," Cancer Research, vol. 77, no. 11, pp. 2976-2989, 2017.

[11] A. Alqahtani, K. Choucair, M. Ashraf et al., "Bromodomain and extra-terminal motif inhibitors: a review of preclinical and clinical advances in cancer therapy," Future Science OA, vol. 5, no. 3, Article ID FSO372, 2019.

[12] G. Andrieu, A. C. Belkina, and G. V. Denis, "Clinical trials for BET inhibitors run ahead of the science," Drug Discovery Today: Technologies, vol. 19, pp. 45-50, 2016.

[13] S. A. Piha-Paul, J. C. Sachdev, M. Barve et al., "First-in-human study of mivebresib (ABBV-075), an oral pan-inhibitor of bromodomain and extra terminal proteins, in patients with relapsed/refractory solid tumors," Clinical Cancer Research, vol. 25, no. 21, p. 6309, 2019.

[14] B. A. Jonas and D. A. Pollyea, "How we use venetoclax with hypomethylating agents for the treatment of newly diagnosed patients with acute myeloid leukemia," Leukemia, vol. 33, no. 12, pp. 2795-2804, 2019.

[15] C. D. DiNardo, K. Pratz, V. Pullarkat et al., "Venetoclax combined with decitabine or azacitidine in treatment-naive, elderly patients with acute myeloid leukemia," Blood, vol. 133, no. 1, pp. 7-17, 2019.

[16] A. H. Wei, S. A. Strickland, J.-Z. Hou et al., "Venetoclax combined with low-dose cytarabine for previously untreated 
patients with acute myeloid leukemia: results from a phase Ib/ II study," Journal of Clinical Oncology, vol. 37, no. 15, pp. 1277-1284, 2019.

[17] E. C. Vamvakas and M. A. Blajchman, "Transfusion-related mortality: the ongoing risks of allogeneic blood transfusion and the available strategies for their prevention," Blood, vol. 113, no. 15, pp. 3406-3417, 2009.

[18] E. Delaflor-Weiss and P. D. Mintz, "The evaluation and management of platelet refractoriness and alloimmunization," Transfusion Medicine Reviews, vol. 14, no. 2, pp. 180-196, 2000.

[19] S. Hosoba, E. K. Waller, N. Shenvi et al., "Peritransplantation red blood cell transfusion is associated with increased risk of graft-versus-host disease after allogeneic hematopoietic stem cell transplantation," Biology of Blood and Marrow Transplantation, vol. 24, no. 5, pp. 973-982, 2018.

[20] M. Mittleman, U. Platzbecker, B. Afanasyev et al., "Eltrombopag for advanced myelodysplastic syndromes or acute myeloid leukaemia and severe thrombocytopenia (ASPIRE): a randomised, placebo-controlled, phase 2 trial," The Lancet Hematology, vol. 5, no. 1, pp. e34-e43, 2018.

[21] K. P. Garnock-Jones and S. J. Keam, "Eltrombopag," Drugs, vol. 69, no. 5, pp. 567-576, 2009.

[22] P. Sepúlveda, A. Encabo, F. Carbonell-Uberos, and M. D. Miñana, "BCL-2 expression is mainly regulated by JAK/ STAT3 pathway in human CD34+ hematopoietic cells," Cell Death \& Differentiation, vol. 14, no. 2, pp. 378-380, 2007.

[23] A. Lollies, S. Hartmann, M. Schneider et al., "An oncogenic axis of STAT-mediated BATF3 upregulation causing MYC activity in classical Hodgkin lymphoma and anaplastic large cell lymphoma," Leukemia, vol. 32, no. 1, pp. 92-101, 2018.

[24] A. Kon, L.-Y. Shih, M. Minamino et al., "Recurrent mutations in multiple components of the cohesin complex in myeloid neoplasms," Nature Genetics, vol. 45, no. 10, pp. 1232-1237, 2013.

[25] J. S. Smith, S. G. Craig, F. G. Liberante et al., "Chronic loss of STAG2 leads to altered chromatin structure contributing to de-regulated transcription in AML," 2019, https://www. biorxiv.org/content/10.1101/856195v1. 